Torreón Universitario www.faremcarazo.unan.edu.ni / ISSN 2410-5708 / e-ISSN 2313-7215

Año 8 | Núm. 21 | pág. 6 - pág. 15 | febrero - mayo 2019
Esta obra está bajo una licencia de Creative Commons

Atribución-NoComercial-SinDerivar 4.0 Internacional

\title{
El posgrado en la UN AN-Managua y su relación con la sociedad nicaragüense II: implicaciones
}

\author{
Dr. Álvaro Antonio Escobar Soriano \\ Universidad Nacional Autónoma de Nicaragua, Managua \\ https://orcid.org/0000-0001-6566-3006 \\ aescobar@unan.edu.ni / alvess007@gmail.com
}

Fecha de recepción: 10 de junio, 2019 / Fecha de aceptación: 25 de octubre, 2019

https://doi.org/10.5377/torreon.v8i21.8850

Palabras clave: posgrado, actualidad, camino recorrido, modernización,

implicaciones.

\section{RESUMEN}

ste artículo expone de manera sintética y objetiva la segunda parte ${ }^{1}$ del análisis
documental que tiene los siguientes objetivos: a) explicitar el desempeño de los
estudios de posgrado de la UNAN-Managua y su relación con la realidad nacional, específicamente con: las instituciones del Estado, la empresa privada y la comunidad, los que tienen vinculación con las funciones sustantivas de la universidad declaradas en sus estatutos y, b) presentar los retos (camino recorrido y por recorrer) y las implicaciones que esto tiene en este subnivel para el futuro de la universidad y del país en general. La descripción actual del posgrado se puede realizar desde varias dimensiones que se relacionan directamente con su quehacer. El conocimiento de estas, pasa por percibir lo mejor posible el impacto social del posgrado desarrollado en la universidad. Por consiguiente, se requiere explicitar y objetivar su desempeño y su relación con la realidad nacional de acuerdo con los ejes: relación con las instituciones del Estado, relación con la empresa privada y relación con la comunidad.

1. En esta segunda parte del estudio se mantienen las referencias bibliográficas de la primera parte, a fin de que el lector tenga clara la idea de que es un solo estudio dividido en dos partes. 


\section{INTRODUCCIÓN}

Este esta segunda parte del estudio documental se expone de manera sintética y objetiva los retos (camino recorrido y por recorrer) y las implicaciones que tiene el posgrado para el futuro de la universidad y del país en general.

Las principales conclusiones a las que se llegó con la primera parte del estudio, la que fue titulada "El posgrado en la UNAN-Managua y su relación con la sociedad nicaragüense I: historia y actualidad" fueron: a) el posgrado en la UNAN-Managua inicia una nueva etapa consolidación y modernización- orientada al ordenamiento y el desarrollo de la investigación y la innovación, b) a fin de obtener valor agregado del conocimiento producido es necesario medir el impacto de la investigación realizada y explorar la factibilidad de desarrollar otras áreas, c) es necesaria la integración de la docencia y la investigación para elevar su efecto en grado y en la realidad nacional y d) falta desarrollar procesos de autoevaluación de los programas, en todas sus categorías.

De esas conclusiones se desprenden implicaciones positivas a mediano y largo plazo: a) en la organización de la universidad, con una nueva estructura en posgrado que aprovecha la visión actual, pero que preparará las bases para una mayor transformación en el próximo quinquenio, b) en la toma de decisiones, de cara a lograr una institución de avanzada en los diferentes campos del conocimiento donde esta tiene fortalezas, y asumir nuevos retos en áreas -en las que hay poca o no se tiene experiencia- en las que se puede invertir y desarrollar, finalmente, c) en relación con el Plan de desarrollo humano, se mejorará: el nivel de formación en los diferentes subsistemas educativos, la producción científica y productiva del país, la capacidad de resolver problemáticas en diferentes niveles, finalmente, el nivel de vida, no solo de los graduados, sino de la población en general.

\section{MATERIAL Y MÉTODOS}

El presente estudio es una investigación documental con una perspectiva histórica. El enfoque con que se desarrolló fue cualitativo. Se realizó el análisis de contenido de diferentes documentos relacionados con la gestión institucional en los que se presentan datos específicos sobre el quehacer del posgrado. Además, se tomó en cuenta documentos de especialistas nacionales e instituciones internacionales como referentes teóricos que sustentan algunos planteamientos y hallazgos.

Las técnicas de recopilación de la información fueron: la revisión bibliográfica, la lista de cotejo y la comparación. Con la revisión documental, se logró el rastreo y localización de las fuentes bibliográficas que aportaron los datos para el estudio. La lista de cotejo (contenía las categorías de análisis que se buscaban en cada documento), se utilizó para clasificar los 
informes de acuerdo con los períodos de tiempo en que fueron elaborados, lo que permitió la reconstrucción de dos etapas: inicial y de expansión difusa.

La comparación permitió el análisis de los hallazgos en relación con las perspectivas teóricas abordadas. Otra dimensión, que permitió desarrollar esta técnica, fue la coherencia entre los datos y la narrativa que se configuró a partir de las descripciones encontradas en los diferentes informes sobre el objeto de estudio.

Con la aplicación de cada una de las técnicas, se obtuvo datos que reflejan aspectos significativos para la comprensión del quehacer del posgrado tanto en su dimensión histórica como en la actual.

El análisis de la información se realizó por períodos, esto dio como resultado la estructuración de un informe crítico-reflexivo que condujo a conclusiones y recomendaciones (implicaciones), que permiten no solo tener una visión sintética del quehacer del postgrado, sino además perspectivas de mejora para el futuro cercano.

\section{RESULTADOS}

\subsection{La autoevaluación y la evaluación con fines de mejora}

Además del reconocimiento nacional, los programas de posgrado necesitan el reconocimiento de su calidad a nivel internacional. Por tanto, es preciso que este se busque a nivel regional en agencias acreditadoras, a las que la institución tenga acceso como miembro, pero también socio. Esto es así, porque el desarrollo de este subsistema ha tenido características muy particulares, diferentes al experimentado en otras universidades del área centroamericana y latinoamericana ${ }^{2}$.

A pesar del desarrollo que se ha venido alcanzando, en posgrado en el último quinquenio, en este tema se ha avanzado muy poco. Por tanto, se ha instaurado la necesidad de dar pasos decisivos en el inicio de procesos de autoevaluación interna de los programas, con el fin de: a) obtener experiencia valiosa en autoevaluación de programas, b) ahorrar fondos a la institución, porque en esta ya existe una masa crítica suficiente para realizar esta acción, y c) iniciar un reordenamiento desde los programas hasta el sistema como tal.

\subsection{Los graduados, la oferta y la demanda de estudios de posgrado}

La cantidad de graduados de posgrado en al III trimestre de este año es de 421, número que fácilmente se elevará a más de seiscientos al finalizar el año académico. De estos, una buena 2. Se debe considerar, por un lado, que hasta finales de la década de los 90 s, CNU logró un acuerdo con el gobierno de turno, dando al fin una salida a una década de confrontaciones y tensiones, efectos de la postguerra civil nicaragüense (Tünnermann y Yarzábal, 2002). Por otro lado, es con la llegada del FSLN al poder a finales de la década pasada, que las universidades del CNU, entre ellas la UNAN-Managua empiezan a recibir mayor atención y recursos. Por tanto, el desarrollo de la misma inicia a inicios de la presente década y a inicios de este último quinquenio la atención se empieza a poner, con mayor énfasis, en el nivel de posgrado. 
cantidad son graduados de la institución. A juzgar por las estadísticas de este año, en su mayoría serán de programas de maestría, seguidos de las especializaciones médicas, programas en los cuales la mayoría de graduados fueron del sexo femenino.

Este sector es importante y se debe tomar muy en serio por la institución, debido al aumento significativo que ha experimentado en este subnivel de formación. Por tanto, los graduados de posgrado deben ser vistos como la mayor representación científica de la UNANManagua, los mejores jueces de la calidad de la formación ofrecida y la mejor fuente de la relación universidad «-» empresa privada, a un nivel de relevancia académica-científica y económica, en especial si se valora que son fuente de creación de conocimiento capaz de transferirse al costo y precio de los productos. (Lage, 2015).

En el Informe de verificación externa (2014), CNEA emitió una serie de recomendaciones para sector graduados de las que, se infiere, a posgrado le corresponden en orden de importancia y desarrollo temporal: a) implantar un sistema informático que además de darles seguimiento, brinde oportunidades de empleo y haga una valoración del impacto del proceso de formación en el campo laboral, b) fomentar procesos de mutua cooperación entre la institución y los graduados posgrado $y, c)$ aplicar pruebas de verificación de competencias para los mismos. A pesar de esto, a nivel de posgrado, este componente no ha tenido la atención necesaria. Por ello, en el contexto de los procesos de autoevaluación con fines de mejora y acreditación de los programas de posgrado, el seguimiento a graduados y la atención a la demanda de programas, es una necesidad de mucha relevancia.

\subsection{Acercamiento al impacto social del posgrado desarrollado en la UNAN-Managua}

La descripción del momento actual que atraviesa el posgrado, arriba expresado, pasa por tener conocimiento objetivo del impacto social. De acuerdo con el Informe de verificación externa (2014, pp. 112 y 113): "La oferta educativa se encuentra en correspondencia con las necesidades de desarrollo socioeconómico del país, de igual manera los perfiles de egreso de los programas de estudio están en correspondencia con las necesidades económicas, sociales y culturales del país". Pero en el ámbito de posgrado se deben hacer algunas observaciones.

La formación continua y profesionalizante -diplomados de formación profesional, especializaciones profesionales, maestrías profesionales- es el grupo de posgrados con mayor demanda. A estos ingresan, por iniciativa propia, personas naturales (en su mayoría provenientes de instituciones privadas) y funcionarios de instituciones del Estado. La oferta es variada y su matrícula tiene un promedio de 20 estudiantes por programa. Por tanto, el impacto en la atención a la demanda es suficiente. Sin embargo, sobre el impacto, en relación con el desarrollo profesional del graduado, aún no se tienen datos que permitan hacer un balance de ello. 
Las especializaciones médico-quirúrgicas tienen una oferta fija para el país. En relación con la matrícula, son el segundo grupo de programas con mayor demanda. No obstante, de acuerdo con datos del MINSA la población de especialistas en las diferentes áreas ofertadas aún es insuficiente para una población creciente, que demanda mejor atención médica especializada.

En el caso de las maestrías académicas y doctorados, en relación con la producción científica en diferentes áreas y tipos de investigación, se puede expresar: existen en la actualidad 15 programas de maestrías académicas y 12 programas de doctorado. Sin embargo, la productividad científica y la publicación todavía son bajas. Además, la calidad de esta no se está midiendo en relación con las áreas en que se desarrolla, la transferencia de conocimiento en la misma institución (a diferentes niveles), y el impacto que pueda tener en los sectores sociales, productivos y científicos donde se pueda aplicar.

\subsection{Los estudios de posgrado de la UNAN-Managua y su relación con la realidad nacional}

Si se considera lo planteado en el acápite anterior, se requiere explicitar y objetivar el desempeño de los estudios de posgrado de la UNAN-Managua y su relación con la realidad nacional de acuerdo con los ejes: relación con las instituciones del Estado, relación con la empresa privada y relación con la comunidad, los que tienen vinculación con las funciones sustantivas de la universidad declaradas en sus estatutos.

En relación con las instituciones del Estado, a partir de la llegada del FSLN al gobierno, la UNAN-Managua ha mejorado sustancialmente su relación con las instituciones. Atiende, a través de diferentes programas de diplomados profesionalizantes y maestrías profesionales (Programa Especial SINACAN) a un número considerable de funcionarios, en campos relacionados con el quehacer de las municipalidades, la producción agrícola, la administración pública, la generación de energías renovables, salud, educación, entre otros.

La relación con la empresa privada es menor. No obstante, en los últimos años, dentro de las políticas de desarrollo y consenso entre el Gobierno de Nicaragua y este sector, se desarrollaron acciones encaminadas a aumentar y fortalecer una visión de trabajo conjunta entre las universidades del CNU y las empresas asociadas a COSEP: conferencias, simposios ferias científicas en campos como innovación, emprendimiento y tecnología. Aún no se han establecido rutas para la formación de personal empresarial y desarrollo conjunto de tecnología. No obstante, con los acontecimientos del 2018, la relación con la empresa privada dio un giro importante hacia el desarrollo y fomento de la Mediana y Pequeña empresa, a fin de potenciarla. Esto también cambió la visión de la universidad, la cual ha empezado a fortalecer la relación con este sector empresarial. 
La comunidad es el sector donde la UNAN-Managua tiene mayor relación. Esto lo hace a través de tres tipos de relaciones: a) la comunidad estudiantil de posgrado realiza sus trabajos de investigación en las comunidades o en centros del Estado que atienden directamente a la población del país, b) trabajos de investigación de programas solicitados por instituciones gubernamentales y c) proyectos de investigación desarrollados desde los centros, institutos y laboratorios especializados.

\subsection{Retos del posgrado: el camino recorrido y por recorrer}

Desde el año 2014, con la aprobación de los Estatutos de la universidad, esta ha venido experimentando un cambio organizacional. En relación con el posgrado, se creó la Dirección de Docencia de Posgrado (DDP) -bajo la orientación del Vicerrectorado de Docencia- la que está dinamizando, el funcionamiento del Sistema de Posgrado, y a la vez, participa en acciones coordinadas con la Dirección de Investigación de Posgrado, entre otras instancias. Sin embargo, es necesaria una mayor integración de todas las estructuras del sistema.

Otro aspecto importante es la creación del Sistema de Seguimiento a Graduados y Pertinencia de la Oferta del Posgrado. Este permitirá la recopilación de información de los graduados, a fin de dar seguimiento a los mismos. Hasta el momento, una comisión ad hoc conformada por especialistas del Vicerrectorado de Docencia han realizado pilotajes de seguimiento, acercamiento a empleadores y un avance sustancial en el documento que regirá la constitución del mismo. Se espera que a mediados de 2018 este sistema pueda empezar a favorecer la obtención de información confiable sobre: la situación de los graduados y su desempeño, su opinión sobre la calidad de la formación realizada; de los empresarios, sobre el desempeño de los anteriores y; de ambos sectores, la percepción sobre la demanda de programas tanto de los que se ofertan como de la emergencia de nuevos.

En relación con los ejes de innovación y emprendimiento -desde una visión responsable, la UNAN-Managua, en aras de fomentar el empoderamiento de la población, en relación con el desarrollo social y en estrecha coordinación con el Gobierno Central- desde posgrado se está preparando la inserción de estos en el currículo de los programas. Estos a su vez, se vincularán con el programa "Aprender, emprender y prosperar", que se desarrolla desde la educación inicial. Estas líneas en posgrado se convertirán en competencias, que los graduados deben desarrollar, lo que apunta a consolidar una cultura de innovación y emprendimiento responsable con el ecosistema nacional, regional y mundial.

La formación para la educación virtual a nivel de posgrado es otro aspecto que se está impulsando. En la actualidad, se ha creado la Dirección de Virtualización, la que se encarga de trasladar programas presenciales a la educación en línea. Con el fin de consolidar la cultura de la educación a distancia virtual, se ha elaborado la "Especialidad en mediación para el aprendizaje 
en la modalidad de educación a distancia virtual", la que será un requisito para los docentes investigadores que incursionen en esta modalidad de trabajo en el posgrado.

El Consejo Superior Universitario Centroamericano (CSUCA) en 1998, inició a desarrollar en Centroamérica, a través del Sistema de Carreras y Posgrado Regionales (SICAR) una estrategia de institucionalización de evaluación y acreditación de programas de posgrado, en la que UNANManagua se incorporó, con el fin de capacitar a su personal.

En el contexto del PEI 2015-2019, y del desarrollo efectivo de las acciones planificadas, se ha elaborado una guía metodológica de autoevaluación (GMA), basada en estándares internacionales como los de ACAP y AUIP, pero con una visión propia que permita mejorar la gestión de los programas de posgrado y elevar, al mayor nivel, la formación.

Con esta guía, se iniciará el proceso de autoevaluación de los programas de posgrado. Este tiene como fines: obtener experiencia en procesos de autoevaluación propios, pero con estándares internacionales, involucrar al personal con mayor nivel a este proceso, dar mayor incidencia a la DDP como garante de la calidad de los procesos, impulsar y facilitar de manera eficiente procesos de evaluación y acreditación internacional, de los programas que hayan alcanzado los estándares requeridos por la GMA y finalmente fortalecer la cultura de evaluación en posgrado.

\subsection{Una nueva organización: hacia la sistematicidad y mejor funcionalidad}

Para lograr un mejor funcionamiento en el Sistema de Estudios de Posgrado, (SEP) es necesario iniciar un proceso de reorganización. Para ello, se ha realizado una propuesta de normas que favorecerá a mediano plazo la consolidación y mejoramiento del posgrado en la UNAN-Managua:

- El Reglamento de Posgrado, como eje central, plantea una nueva organización de los programas, en especial aquellos orientados a la formación y producción científica.

- La Normativa del funcionamiento del Sistema de Estudios de Posgrado, recoge la experiencia de la organización actual del sistema, como apoyo para la conformación de las Comisiones Académicas Científicas en facultades, centros e institutos de investigación. Esta estructura se convertirá en el soporte para una transformación a mayor profundidad en el próximo quinquenio. Con esta, se espera consolidar los programas y a su vez la investigación, porque será la instancia que garantizará -entre otras funciones- el desarrollo de las líneas de investigación, a través de la creación y legalización de grupos de investigación. (Política de Investigación e Innovación, 2017).

- Con la Normativa de estudios de formación continua, se espera poder enlazar de manera efectiva este nivel de formación con los estudios de posgrado. 


\section{CONCLUSIONES E IMPLICACIONES}

Desde cada uno de los aspectos abordados en esta segunda parte del trabajo se puede concluir que de acuerdo con el cumplimiento que se vaya logrando en el desarrollo de este subnivel de la educación superior, se tendrán las siguientes implicaciones positivas, a mediano plazo, en:

- La organización de la universidad, porque a mediano plazo los programas se consolidarán en temas como la gestión, la investigación y la autoevaluación. Una vez se consoliden estos procesos, y a largo plazo, se podrá reestructurar el sistema, de manera que se alcance una nueva estructura orientada a la conformación de centros o escuelas de posgrado -siguiendo la tendencia internacional- o una nueva estructura semiautónoma que favorezca el desarrollo pleno de la investigación, docencia, gestión e internacionalización del posgrado de la UNAN-Managua.

- La funcionalidad del sistema en varios aspectos. Sin embargo, es urgente mejorar en: a) sistema de ingreso, matrícula, registro y apertura de los programas, los que deben ajustarsealcalendarioacadémico delauniversidad,b)retención, promociónygraduación, con el seguimiento de los programas mediante una estrategia de acompañamiento académico-científico comprometido de parte de todas las estructuras de posgrado, en especial de los coordinadores y responsables facultativos, c) el compromiso de los profesores nacionales y extranjeros a participar genuinamente con los programas tanto en la docencia, en la tutoría y en la investigación, mediante instrumentos de contratación explícitos y d) en la administración -mancomunada entre administración de facultades centros o institutos de investigación y coordinaciones de programas en la mejora de medios, locales, atención a estudiantes y docentes - de los fondos generados por los programas, con el control debido de auditoría interna de la institución.

A mediano y largo plazo, respectivamente, en:

- El análisis sistemático y objetivo del posgrado en la UNAN-Managua, como procesos que traerá consigo cambios sustanciales en las decisiones que se tomen en relación con el quehacer de este nivel de la educación superior, de cara a lograr una institución de avanzada en los diferentes campos del conocimiento donde esta tiene fortalezas, y asumir nuevos retos en áreas -en las que hay poca o no se tiene experiencia - en las que se puede invertir y desarrollar.

- La extensión de la universidad y su impacto en la sociedad en relación con el Plan de desarrollo humano, porque se mejorará: el nivel de formación en los diferentes subsistemas educativos, la producción científica y productiva del país, la capacidad de 
resolver problemáticas no solo a nivel local, sino a nivel nacional y finalmente el nivel de vida, no solo de los graduados, sino de la población en general.

\section{REFERENCIAS BIBLIOGRÁFICAS}

Consejo Nacional de Evaluación y Acreditación Institucional. (2014). Informe de verificación externa UNANManagua. Managua: CNEA

Consejo Nacional de Universidades. (2004). Informe de posgrado CNU. Managua: CNU.

Consejo Nacional de Universidades. (2005). Informe de posgrado CNU. Managua: $\mathrm{CNU}$.

Consejo Nacional de Universidades. (2006). Informe de posgrado CNU. Managua: CNU.

Consejo Nacional de Universidades. (2008). Informe de posgrado CNU. Managua: CNU.

Consejo Nacional de Universidades. (2009). Informe de posgrado CNU. Managua: CNU. Consejo Nacional de Universidades. (2011). Informe de posgrado CNU. Managua: CNU. Consejo Nacional de Universidades. (2013). Informe de posgrado CNU. Managua: CNU. Consejo Nacional de Universidades. (2015). Informe de posgrado CNU. Managua: CNU.

Escobar, A. (2017). Retos del posgrado en la UNAN-MANAGUA y sus implicaciones en la sociedad nicaragüense. Managua: UNANManagua.

Lage, A. (2015). La economía del conocimiento y el socialismo. Preguntas y respuestas. Habana: Academia.
Organization for Economic Co-operation and Development (OECD). (2002). Manual de Frascati. Madrid: Fundación Española para la Ciencia y la Tecnología.

Rocha, L. (junio 20, 2017). Graduados en programas de maestría y ciencias médicas. Inscripción en registro académico. Serie de datos completa 2007-2015 (incluye abril 2016). Managua: UNAN-Managua.

Tünnermann, C y Yarzábal, L, (2002). Tendencias y potencialidades del desarrollo de la educación superior en Nicaragua. Managua: Asdi.

Universidad Nacional Autónoma de Nicaragua Managua, UNAN-Managua. (2011). Reglamento del Sistema de Estudios de Posgrado y Educación Continua, SEPECUNAN-Managua. Managua: UNANManagua.

Universidad Nacional Autónoma de Nicaragua Managua, UNAN-Managua. (2017). Plan operativo anual institucional, 2017. Managua: UNAN-Managua.

Universidad Nacional Autónoma de Nicaragua Managua, UNAN-Managua. (2017). Plan de monitoreo, seguimiento y evaluación del Plan estratégico institucional 2015 - 2019. Managua: UNAN-Managua. 
Universidad Nacional Autónoma de Universidad Nacional Autónoma de Nicaragua, Managua UNAN-Managua. (2017). Informes físico-financieros. I, II y III trimestre 2017. Managua: UNANManagua.

Universidad Nacional Autónoma de Nicaragua, Managua UNAN-Managua. (agosto, 2017). Informe, indicadores de investigación y posgrado de la UNANManagua solicitado por CSUCA (2016). Managua: UNAN-Managua.

Universidad Nacional Autónoma de Nicaragua, Managua UNAN-Managua. (2014). Estatutos. Managua: UNANManagua.

Nicaragua, Managua UNAN-Managua. (2016). Informe físico-financiero anual 2016 de la Unan-Managua. Managua: UNAN-Managua.

Universidad Nacional Autónoma de Nicaragua, Managua UNAN-Managua. (2017). Política de Investigación e Innovación, 2017. Managua: UNANManagua. 